\title{
Paper Developing RF Power Sensor Calibration Station in Direct Comparison Transfer System using Vector Network Analyzer
}

\author{
Jarosław Szatkowski
}

National Institute of Telecommunications, Warsaw, Poland

https://doi.org/10.26636/jtit.2021.155021

\begin{abstract}
Calibration of RF power sensors is crucial issue in RF power measurements. Many calibration laboratories use the direct comparison transfer system with a signal generator and a power splitter. Increasing performance of modern vector network analyzers makes it possible to perform a power sensor calibration with acceptable uncertainties. The main advantage when using a VNA is a simple measurement setup with a wide frequency range (up to $50 \mathrm{GHz}$, limited only by the VNA and the standard power sensor), where all of required components, i.e. signal generator, a directional coupler and a reference power indicator are built in the VNA technology. This paper reports performing a VNA-based RF power sensors calibration for $10 \mathrm{MHz}-18 \mathrm{GHz}$ band, carried out in the Laboratory of Electric, Electronic and Optoelectronic Metrology at the National Institute of Telecommunications in Warsaw, Poland. In order to validate the proposed solution two of power sensors were calibrated at a reference laboratory. The validation consisted of two steps. At first, one of those characterized power sensors was calibrated at our laboratory in direct comparison transfer system. Finally, the results obtained from the VNA-based system were compared with the previously obtained ones.
\end{abstract}

Keywords-direct comparison transfer, microwave power measurements, power sensor calibration, measurement uncertainty, VNA.

\section{Introduction}

Measurement of RF signal power is one of the most important metrology issues in microwaves. Power sensors together with suitable power meters are used in test stand. To achieve best accuracy of the measurement, a standard power sensor is calibrated by one of the national laboratory, for instance NIST, METAS, PTB, etc. For power sensor calibration the direct comparison transfer method is widely used [1], [2]. The calibration system consists of a power splitter or a directional coupler where input port is connected to signal generator, and have a reference power sensor attached to one of the two output ports as shown in the Fig. 1. The second output port is connected to the stan- dard power sensor and to the power sensor being calibrated (device under test, DUT) interchangeably. The calibration performance depends on the effective source match of the splitter or the directional coupler, and on the accuracy of the used standard power sensor.

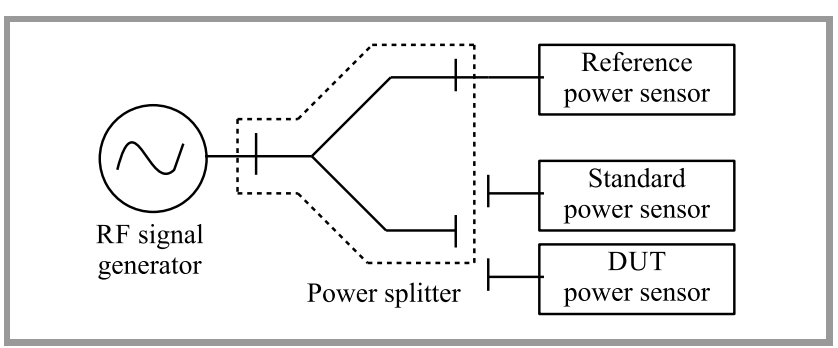

Fig. 1. Power sensor calibration system in generator - splitter connection for direct comparison transfer.

The use of vector network analyzer (VNA) for calibration power sensors was presented in [3], [4]. The principle of operation of the VNA-based system is same as for generator-splitter method, hence it is the direct comparison transfer system with identical sources of measurement uncertainty. However, the quality of VNA output signal is generally much worse than that of the signal obtained from RF generators, hence it can significantly affect the uncertainty budget. A comparison of these two systems was presented in [4]. The main benefit of using the VNA versus the generator-splitter system is its great simplicity. Figure 2 shows the block diagram of VNA port 1.

The standard power sensor and the unit to be calibrated are alternately connected to the VNA. The signal generator, the directional coupler, and the reference power sensor are circuits built into VNA, thus the setup of the system is very simple.

This paper presents the VNA-based power sensor calibration system for frequency range $10 \mathrm{MHz}-18 \mathrm{GHz}$. This is a preliminary work proving the concept of VNA usage for this purpose. The aim is to set up the measurement station covering the VNA full range, up to $50 \mathrm{GHz}$. 


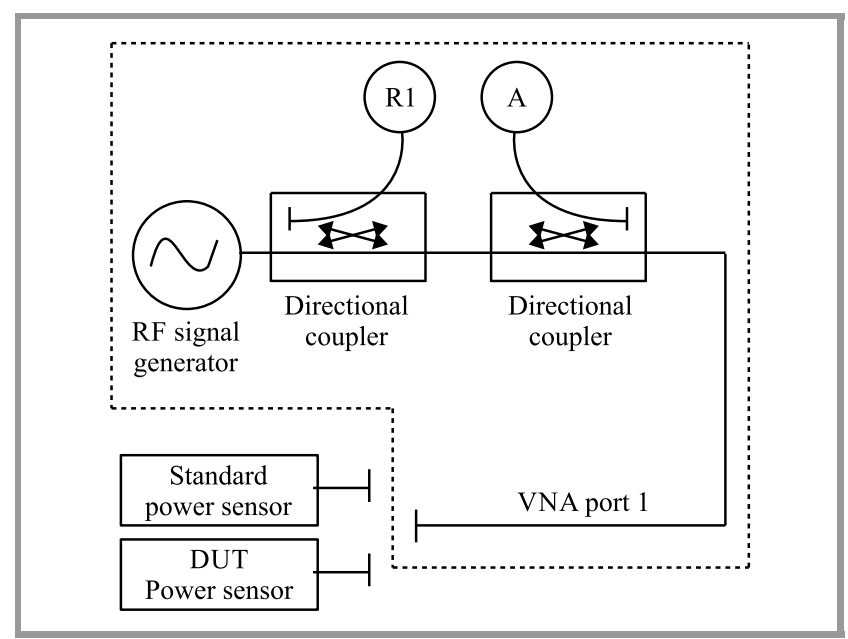

Fig. 2. Block diagram of VNA port 1.

In order to validate the developed system two power sensors were calibrated in a reference laboratory. One of them was then used as the standard power sensor, whereas the second one was used as the DUT. The results retrieved from the reference lab and the data obtained with the generatorsplitter method and with the VNA system were compared.

\section{Direct Comparison Transfer Overview}

The measurement system consists of a RF signal generator, a power splitter (or directional coupler), and a reference power sensor connected to one of the output ports of the splitter. The standard power sensor and DUT are alternately connected to the second port of the splitter (see Fig. 1). Using the reference power sensor and the power splitter instead of connecting the sensors directly to the output of the generator improves the effective source match. The effective source match $\Gamma_{e 2}$ depends only on the scattering parameters of the power splitter itself and is generally much better and stable than the source match of the generator [5]. Importantly, it is quite easy to measure using VNA, either by determining the scattering parameters or by Jureshek method [6]. In contrast, source match of signal generators is quite difficult to determine:

$$
\Gamma_{e 2}=S_{33}-\frac{S_{31} S_{23}}{S_{21}}
$$

The DUT calibration factor $K_{D}$ is calculating using [4]:

$$
K_{D}=K_{S} \frac{P_{D}}{P_{M D}} \cdot \frac{P_{M S}}{P_{S}} \cdot\left(\frac{\left|1-\Gamma_{e 2} \Gamma_{D}\right|}{\left|1-\Gamma_{e 2} \Gamma_{S}\right|}\right)^{2},
$$

where $P_{S}, P_{M S}, P_{D}, P_{M D}$ are power levels measured by the standard power sensor, by the reference power sensor with the standard sensor connected, by the DUT power sensor, and by the reference power sensor with the DUT sensor connected, respectively. $\Gamma_{S}$ and $\Gamma_{D}$ are the reflection coefficients of the standard and of the DUT. The B type uncertainty of the measured calibration factor $K_{D}$ mainly consists of the uncertainty of the transferred calibration factor $K_{S}$ and the uncertainties of the reflection coefficients in the mismatch factor in Eq. 2. It is worth noting, that the calibration factor $K_{D}$ does not depend on the calibration factor and the reflection coefficient of the reference power sensor, thus this power sensor does not affect the B type calibration uncertainty. The A type uncertainty mainly includes power readings instabilities of each power sensor used, as well as the connection repeatability. The uncertainty budget for a calibration of a power sensor with the direct comparison transfer method is described in [1].

\section{VNA in Power Sensor Calibration}

A vector network analyzer is used for a measurement of a reflection of a calibrated power sensor. It is a very important parameter specified by all manufacturers of power sensors. In addition, reflection coefficients of standard and DUT power sensors are required to calculate the calibration factor $K_{D}$ by Eq. (2) and are taking into account when calculating measurement uncertainties.

This article shows the usage of a VNA in a direct comparison transfer system to determine the calibration factor $K_{D}$. Furthermore, VNA can be used to calibrate the linearity factor, thus it makes it possible to perform a complete power sensor calibration in a simple way [3].

The VNA integrates all required components for measurement of the calibration factor with the direct comparison transfer system. When looking at Fig. 2 and comparing it with Fig. 1 one can clearly deduce that the directional coupler with R1 receiver works the same way as the power splitter with the reference power sensor. The signal generator is built into VNA. Thus the formula for the calibration factor of DUT sensor is similar to the Eq. (2):

$$
K_{D}=K_{S} \frac{P_{D}}{\left|R 1_{D}\right|^{2}} \cdot \frac{\left|R l_{S}\right|^{2}}{P_{S}} \cdot\left(\frac{\left|1-\Gamma_{e s} \Gamma_{D}\right|}{\left|1-\Gamma_{e s} \Gamma_{S}\right|}\right)^{2},
$$

where $R l_{D}$ and $R l_{S}$ are the measured complex incident voltage waves when the standard power sensor and the DUT are connected to the VNA port, respectively, and $\Gamma_{e s}$ is the source match of the port used. $\Gamma_{e s}$ is relatively easy to determine as it is one of the error terms retrieved from reflection calibration of the VNA port [7]. The similarity of Eqs. (2) and (3) reflects in the uncertainty analysis, where uncertainty factors similar to those in the generator-splitter method are taken into account [7], [8].

\section{Measurements}

\subsection{Measurement Setups}

The DUT and standard power sensors were respectively a diode power sensor and a thermal power sensor manufactured by Keysight Technologies. Both power sensors had been calibrated in a reference laboratory at frequencies ranging from $10 \mathrm{MHz}$ to $18 \mathrm{GHz}$. Figure 3 presents the 


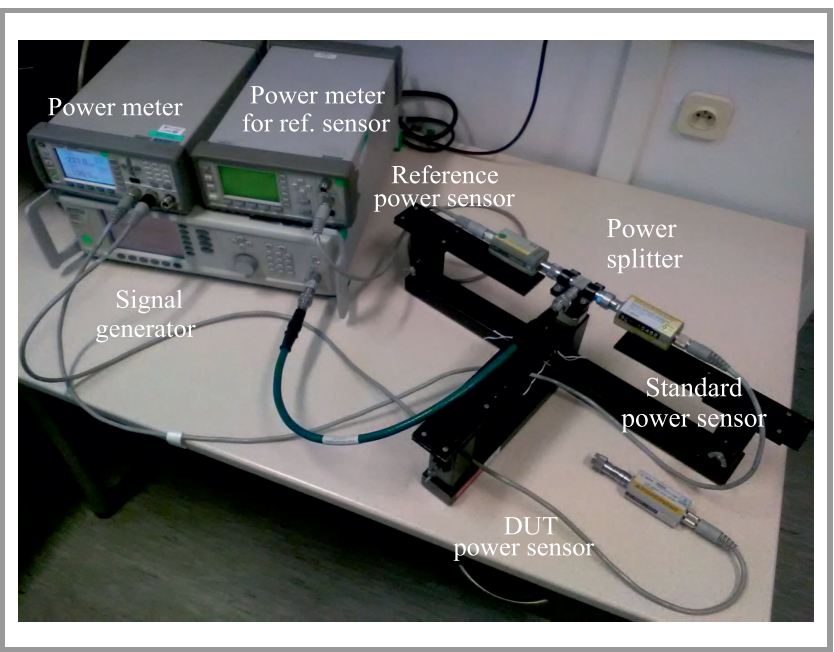

Fig. 3. Measurement setup with a signal generator, a power splitter and with a reference power sensor for power sensor calibration in direct comparison transfer system.

calibration system setup with a signal generator, a power splitter and a reference power sensor. The VNA-based system with port 1 involved is shown in Fig. 4. Both circuits consisted of a standard and a DUT power sensors, as well as a power meter for power readings from the sensors. The generator-splitter setup in addition to a signal generator and a power splitter has a reference power sensor with its corresponding power meter. It is evident from the photos that using the system with a VNA requires less equipment, although is more expensive. However, as mentioned earlier, the VNA is necessary for power sensor calibration and most likely it is already present in the calibration laboratories.

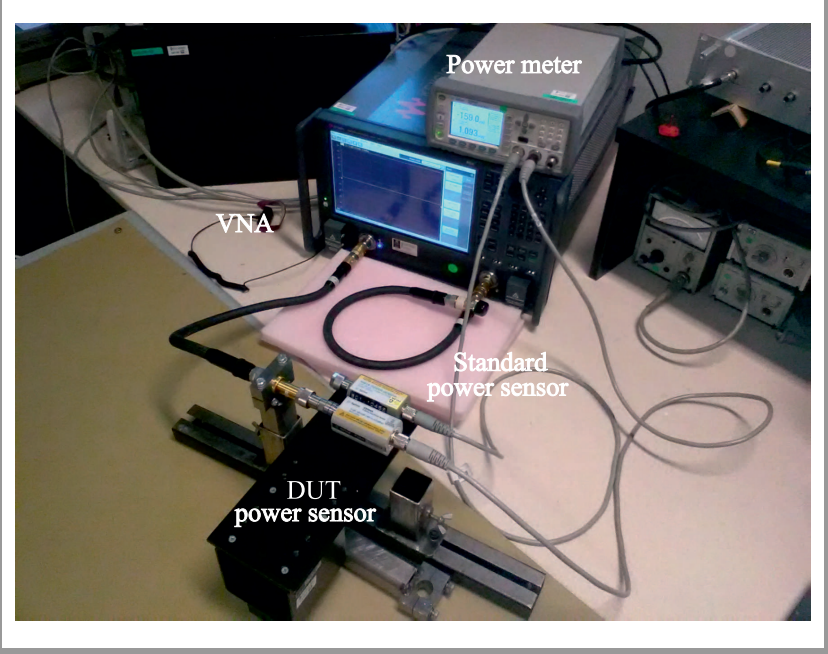

Fig. 4. VNA-based measurement system for power sensor calibration in direct comparison transfer system.

\subsection{Source Match Evaluation}

First, the source reflections at the splitter output and at port 1 of the VNA with cable were evaluated. With this purpose an electronic calibration unit was used. The source match characterization of the splitter was performed using the Juroshek method [6]. The source match of the VNA system was retrieved as an error term after oneport reflection calibration [9]. Figure 5 shows the results of both source reflection measurements. The equivalent source match of the power splitter is better than the source match of the VNA in the whole frequency range of interest. For the frequencies up to $2 \mathrm{GHz}$ a source reflection of the splitter is extremely low.

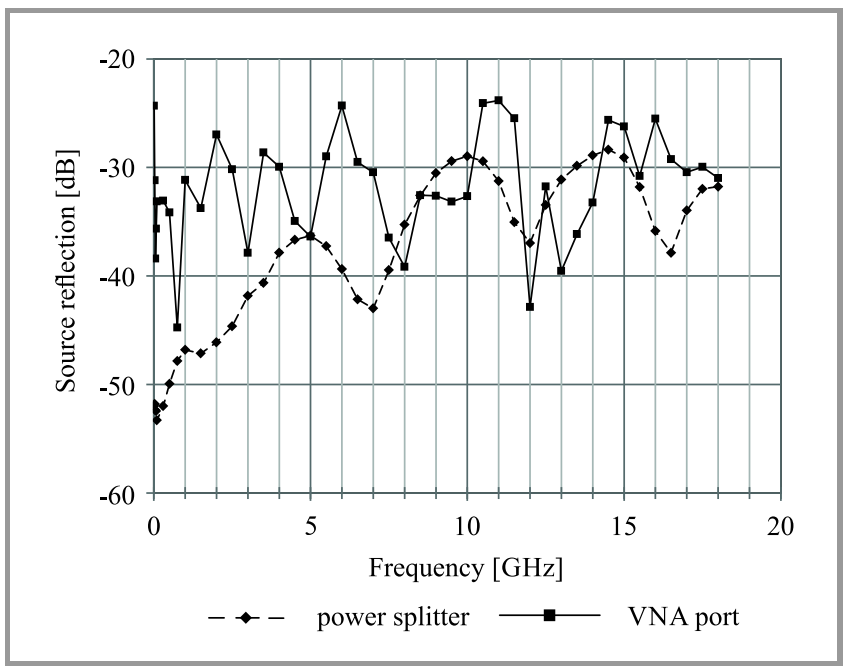

Fig. 5. Source reflections of the power splitter and VNA port 1 with cable.

\subsection{Calibration Results}

The results of power sensor calibration using a generatorsplitter setup, using a VNA-based system, and those obtained from the reference lab are depicted in Fig. 6. Corresponding data for both systems are presented in Table 1. Results from a generator-splitter system seem a bit more consistent with results from the reference laboratory. Discrepancies are especially noticeable around $10 \mathrm{GHz}$. In order to evaluate the performance of the VNA system a figure of merit $E n$ was calculated [10]:

$$
E n=\left|\frac{K_{D, A}-K_{D, B}}{\sqrt{U_{A}^{2}+U_{B}^{2}}}\right|,
$$

where $K_{D, A}, K_{D, B}$ are the calibration factor results from the VNA method and from the generator-splitter method, respectively. $U_{A}$ and $U_{B}$ are corresponding expanded uncertainties. Uncertainties for both methods were calculated according to ISO GUM [11], to internal laboratory procedures, and to uncertainty budges presented in [1], [3], [8]. A graph of the En parameter is shown in Fig. 7, and the corresponding data is presented in Table 1. According to [10] the comparison result is accepted when $E n<1$. In this case a maximum value of 0.79 is obtained for $10 \mathrm{MHz}$, which means that the obtained variances are acceptable. 


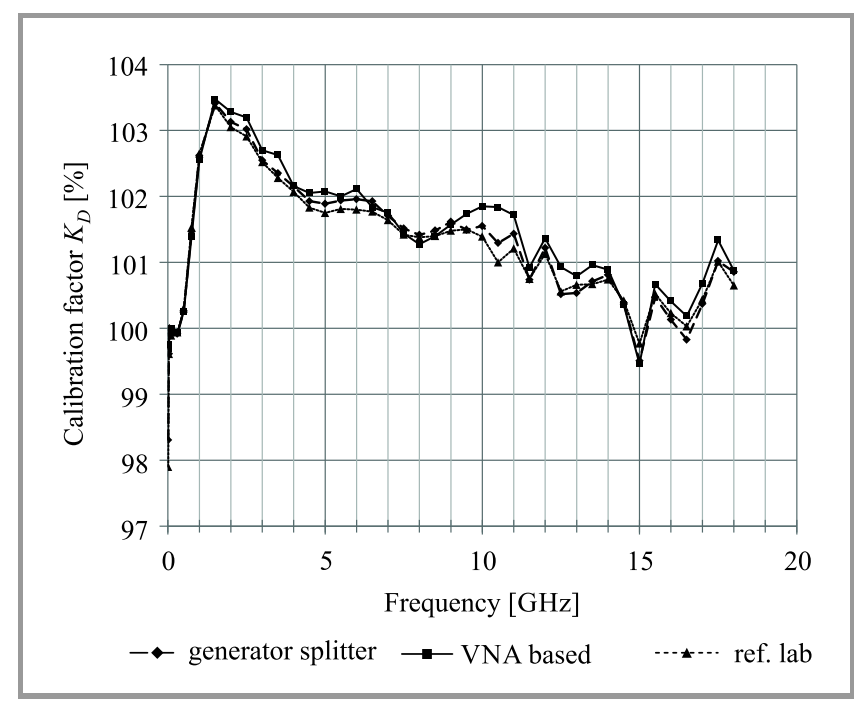

Fig. 6. Calibration factors of DUT power sensor obtained from generator-splitter system, VNA system, and from reference lab.

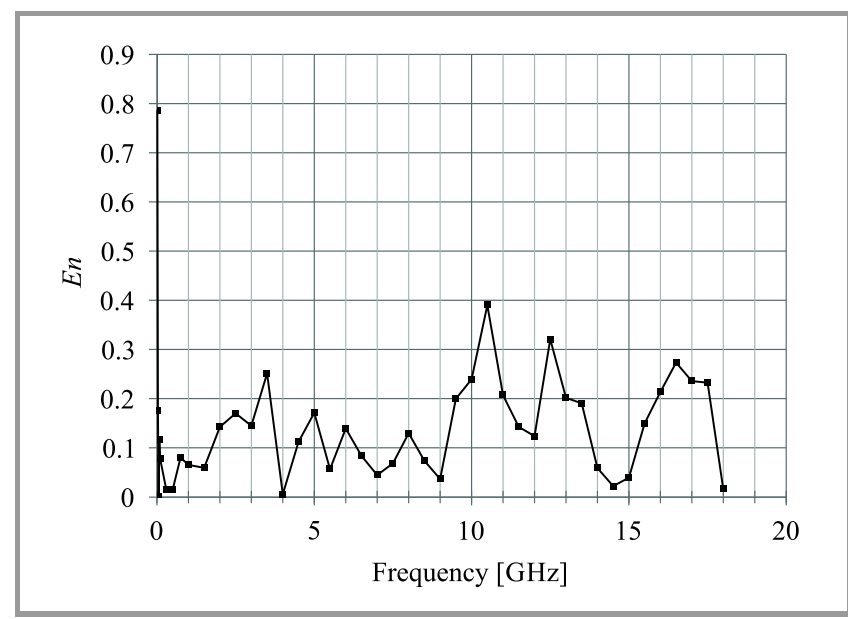

Fig. 7. En parameter for VNA method and generator-splitter method.

Generally quite good consistency is observable between the VNA-based method and the generator-splitter method. Second highest value of $E n$ is 0.39 for $10.5 \mathrm{GHz}$.

\section{Conclusions}

This article presented the development of a RF power sensor calibration setup which makes use of a VNA at frequencies ranging from $10 \mathrm{MHz}$ to $18 \mathrm{GHz}$, in the Laboratory of Electric, Electronic and Optoelectronic Metrology at the National Institute of Telecommunications in Warsaw, Poland. As the preliminary work for full-range measurement capability to calibrate the power sensors up to $50 \mathrm{GHz}$, it proves the concept, that VNA can successfully be used to determine the calibration factor of power sensors in the direct comparison transfer system instead of a wellknown generator-splitter method. The comparison between the two methods showed a good mutual consistency.
Table 1

Power sensor calibration results

\begin{tabular}{|c|c|c|c|c|c|}
\hline Freq. [GHz] & $K_{D . A}[\%]$ & $U_{A}[\%]$ & $K_{D . B}[\%]$ & $U_{B}[\%]$ & En \\
\hline 0.01 & 99.69 & 1.50 & 98.31 & 0.92 & 0.79 \\
\hline 0.03 & 99.76 & 0.61 & 99.61 & 0.57 & 0.17 \\
\hline 0.05 & 100.00 & 0.38 & 100.00 & 0.37 & 0.00 \\
\hline 0.075 & 100.00 & 0.63 & 99.90 & 0.62 & 0.12 \\
\hline 0.1 & 99.99 & 0.63 & 99.92 & 0.62 & 0.08 \\
\hline 0.3 & 99.93 & 0.71 & 99.92 & 0.70 & 0.01 \\
\hline 0.5 & 100.25 & 0.71 & 100.27 & 0.71 & 0.02 \\
\hline 0.75 & 101.39 & 0.72 & 101.47 & 0.72 & 0.08 \\
\hline 1.0 & 102.55 & 0.74 & 102.62 & 0.72 & 0.07 \\
\hline 1.5 & 103.47 & 0.75 & 103.41 & 0.74 & 0.06 \\
\hline 2.0 & 103.28 & 0.76 & 103.13 & 0.73 & 0.14 \\
\hline 2.5 & 103.20 & 0.76 & 103.02 & 0.73 & 0.17 \\
\hline 3.0 & 102.70 & 0.74 & 102.55 & 0.73 & 0.14 \\
\hline 3.5 & 102.63 & 0.81 & 102.35 & 0.73 & 0.25 \\
\hline 4.0 & 102.16 & 0.80 & 102.15 & 0.73 & 0.01 \\
\hline 4.5 & 102.05 & 0.79 & 101.93 & 0.77 & 0.11 \\
\hline 5.0 & 102.08 & 0.78 & 101.89 & 0.76 & 0.17 \\
\hline 5.5 & 102.00 & 0.80 & 101.94 & 0.76 & 0.06 \\
\hline 6.0 & 102.11 & 0.82 & 101.96 & 0.75 & 0.14 \\
\hline 6.5 & 101.84 & 0.77 & 101.93 & 0.76 & 0.08 \\
\hline 7.0 & 101.75 & 0.78 & 101.71 & 0.75 & 0.04 \\
\hline 7.5 & 101.44 & 0.78 & 101.51 & 0.78 & 0.07 \\
\hline 8.0 & 101.27 & 0.78 & 101.42 & 0.79 & 0.13 \\
\hline 8.5 & 101.40 & 0.83 & 101.48 & 0.83 & 0.07 \\
\hline 9.0 & 101.57 & 0.84 & 101.62 & 0.84 & 0.04 \\
\hline 9.5 & 101.74 & 0.85 & 101.49 & 0.87 & 0.20 \\
\hline 10.0 & 101.85 & 0.86 & 101.55 & 0.90 & 0.24 \\
\hline 10.5 & 101.84 & 1.07 & 101.29 & 0.90 & 0.39 \\
\hline 11.0 & 101.72 & 1.06 & 101.43 & 0.87 & 0.21 \\
\hline 11.5 & 100.92 & 0.99 & 100.74 & 0.84 & 0.14 \\
\hline 12.0 & 101.37 & 0.82 & 101.22 & 0.84 & 0.12 \\
\hline 12.5 & 100.93 & 0.91 & 100.52 & 0.90 & 0.32 \\
\hline 13.0 & 100.79 & 0.86 & 100.54 & 0.93 & 0.20 \\
\hline 13.5 & 100.96 & 0.87 & 100.71 & 0.96 & 0.19 \\
\hline 14.0 & 100.89 & 0.89 & 100.81 & 0.98 & 0.06 \\
\hline 14.5 & 100.35 & 1.05 & 100.38 & 0.97 & 0.02 \\
\hline 15.0 & 99.46 & 0.92 & 99.51 & 0.87 & 0.04 \\
\hline 15.5 & 100.67 & 0.93 & 100.47 & 0.93 & 0.15 \\
\hline 16.0 & 100.41 & 0.94 & 100.13 & 0.92 & 0.21 \\
\hline 16.5 & 100.18 & 0.92 & 99.83 & 0.92 & 0.27 \\
\hline 17.0 & 100.68 & 0.94 & 100.37 & 0.94 & 0.24 \\
\hline 17.5 & 101.34 & 0.97 & 101.02 & 0.96 & 0.23 \\
\hline 18.0 & 100.88 & 0.99 & 100.86 & 0.98 & 0.02 \\
\hline
\end{tabular}

\section{References}

[1] M. P. Weidman, "Direct comparison transfer of microwave power sensor calibrations", NIST Technical Note 1379, Washington: 1996 [Online]. Available: https://www.nist.gov/document-14769 
[2] Y. Shan, Y. S. Meng, and Z. Lin, "Generic model and case studies of microwave power sensor calibration using direct comparison transfer", IEEE Transac. on Instrumentat. and Measurement, vol. 62, no. 6, pp. 1834-1839, 2013 (DOI: 10.1109/TIM.2012.2225961).

[3] K. Wong, "Complete power sensor calibration using a VNA", in Proc. 80th ARFTG Microwave Measurement Conf., San Diego, CA, USA, 2012, pp. 1-5 (DOI: 10.1109/ARFTG.2012.6422420).

[4] W. K. P. Angin, J. Kwon, T. Kang, and N. Kang, "Comparison of RF power sensor calibration using a vector network analyzer and a direct transfer system", in 2016 URSI Asia-Pacific Radio Sci. Conf. (URSI AP-RASC), Seoul, Korea (South), 2016, pp. 1754-1756 (DOI: 10.1109/URSIAP-RASC.2016.7601209).

[5] R. A. Johnson, "Understanding microwave power splitters", Microwave J., pp. 49-56, 1975.

[6] J. R. Juroshek, "A direct calibration method for measuring equivalent source mismatch", Microwave J., vol. 40, no. 10, pp. 106-118, 1997.

[7] J. R. Fenton, "Vector-corrected power sensor calibration", in 51st ARFTG Conf. Digest, Baltimore, MD, USA, 1998 (DOI: 10.1109/ARFTG.1998.327286).

[8] F. Aldossary, Z. Huneiti, Z. Hunaiti, and W. Balachandran, "The network analyser (HP8510C) as a transfer instrument for power sensor calibration", in 2008 IEEE Instrument. and Measurement Technol. Conf., Victoria, BC, Canada, 2008, pp. 1249-1253 (DOI: 10.1109/IMTC.2008.4547233).

[9] J. Fitzpatrick, "Error models for systems measurements", Microwave $J .$, pp. 63-66, 1978

[10] ISO 13528, "Statistical methods for use in proficiency testing by interlaboratory comparisons", Int. Organization for Standardization, Geneva, 2005 [Online]. Available: https://www.iso.org/standard/35664.html
[11] "Evaluation of measurement data - Guide to the expression of uncertainty in measurement", Joint Committee for Guides in Metrology, 2008 [Online]. Available: https://www.bipm.org/ documents/20126/2071204/JCGM_100_2008_E.pdf/ cb0ef43f-baa5-11cf-3f85-4dcd86f77bd6

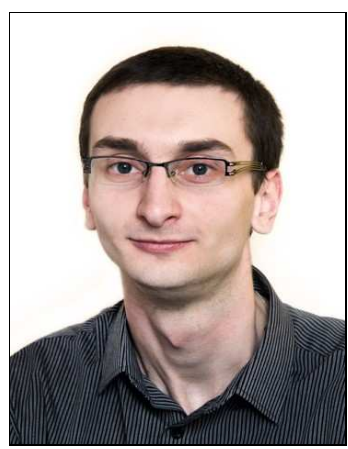

Jarosław Szatkowski received his B.Sc. and M.Sc. in Electronics from Warsaw University of Technology, Poland, in 2015 and 2017 respectively. Since 2017 he is with Laboratory of Electrical, Electronic and Optoelectronic Metrology in $\mathrm{Na}-$ tional Institute of Telecommunications where he is involved in calibration of RF metrology instrumentation. His research interests focus mainly on microwave metrology such as vector network analysis and RF power measurements.

(Di) https://orcid.org/0000-0002-5788-1852

E-mail: j.szatkowski@il-pib.pl

National Institute of Telecommunications

Szachowa 1

04-894 Warsaw, Poland 